\title{
Role of potassium orbitals in the metallic behavior of $K_{3}$ picene
}

\author{
G. Chiappe and E. Louis \\ Departamento de Física Aplicada, Unidad Asociada del CSIC and Instituto Universitario de Materiales, Universidad de Alicante, \\ San Vicente del Raspeig, 03690 Alicante, Spain
}

\begin{abstract}
A. Guijarro
Departamento de Química Orgánica and Instituto Universitario de Síntesis Orgánica, Universidad de Alicante, San Vicente del Raspeig, 03690 Alicante, Spain
\end{abstract}

E. San-Fabián

Departamento de Química Física, Unidad Asociada del CSIC and Instituto Universitario de Materiales, Universidad de Alicante, San Vicente del Raspeig, 03690 Alicante, Spain

\author{
J. A. Vergés* \\ Departamento de Teoría y Simulación de Materiales, Instituto de Ciencia de Materiales de Madrid (CSIC), \\ Cantoblanco, 28049 Madrid, Spain
}

(Received 7 February 2014; revised manuscript received 30 May 2014; published 10 July 2014)

\begin{abstract}
Detailed electronic structure calculations of picene clusters doped by potassium modeling the crystalline $\mathrm{K}_{3}$ picene structure show that while two electrons are completely transferred from potassium atoms to the lowest-energy unoccupied molecular orbital of pristine picene, the third one remains closely attached to both material components. Multiconfigurational analysis is necessary to show that many structures of almost degenerate total energies compete to define the cluster ground state. Our results prove that the $4 \mathrm{~s}$ orbital of potassium should be included in any interaction model describing the material. We propose a quarter-filled two-orbital model as the most simple model capable of describing the electronic structure of K-intercalated picene. Precise solutions obtained by a development of the Lanczos method show low-energy electronic excitations involving orbitals located at different positions. Consequently, metallic transport is possible in spite of the clear dominance of interaction over hopping.
\end{abstract}

DOI: 10.1103/PhysRevB.90.035109

PACS number(s): 71.28.+d, 36.40.Cg, 71.10.Fd, 71.27.+a

\section{INTRODUCTION}

Doping of crystals formed by large polycyclic aromatic hydrocarbon (PAH) molecules such as picene has originated a new class of superconducting materials of very promising characteristics. The pioneering work of Mitsuhashi et al. [1] showed superconductivity of potassium-intercalated picene at 7 or $18 \mathrm{~K}$, depending on sample processing. Later, other PAH crystals formed by coronene [2], phenanthrene [3], and dibenzopentacene [4] have also shown superconducting properties according to the temperature behavior of magnetic susceptibility. Doping with group 2 elements is also possible [5]. Very recently, zero resistivity in K-doped picene has been observed [7].

Parallel to the experimental work there have been several theoretical calculations of the electronic structure of potassium-intercalated picene [8-15]. According to x-raydiffraction experiments, potassium enters into the intralayer herringbone arrangement of picene molecules. The exact position of $\mathrm{K}$ atoms is not known but several density functional theory (DFT) computations agree, locating $\mathrm{K}$ atoms in rows of three atoms that are approximately equidistant to four picene molecules (see the central $\mathrm{K}$ group in the bottom right panel of Fig. 1). In this way, the original structure of pristine crystalline picene is minimally distorted. The relevant

*jav@icmm.csic.es electronic bands are above the original semiconducting gap of undoped picene. They are modified by the presence of potassium and occupied by three electrons coming from doping $\mathrm{K}$ atoms. It is usually assumed that the band formed by the lowest-energy unoccupied molecular orbital (LUMO) of picene is completely occupied, whereas the LUMO+1 band remains half-occupied. This image supports a conducting system that becomes superconducting when the temperature decreases sufficiently. On the other hand, some papers have pointed to a magnetic character of the last bands that invalidates the naive picture of independent electrons $[9,11,15,16]$.

In this paper, we want to emphasize the role of the $4 s \mathrm{~K}$ orbital in the occupied part of the electronic band structure. While our calculations show that two $\mathrm{K}$ electrons go to the picene LUMO, the third $\mathrm{K}$ electron feels almost equal potentials at $4 s$ atomic $\mathrm{K}$ orbital and picene $\mathrm{LUMO}+1$, and consequently, remains in a kind of resonating situation [17]. We suggest that this capability of the last doping electron for visiting both the PAH molecule and the doping site should be the ultimate reason for the metallic behavior of the compound.

The rest of the paper is organized as follows. Section II is devoted to giving some details of the methods and procedures used in this work. The selection and geometry optimization of clusters is presented and standard quantum chemistry methods valid in multiconfigurational cases are briefly described. Section III gives our main quantum chemistry computational results together with some discussion. Section IV presents and discusses a minimal interaction model for the most relevant 


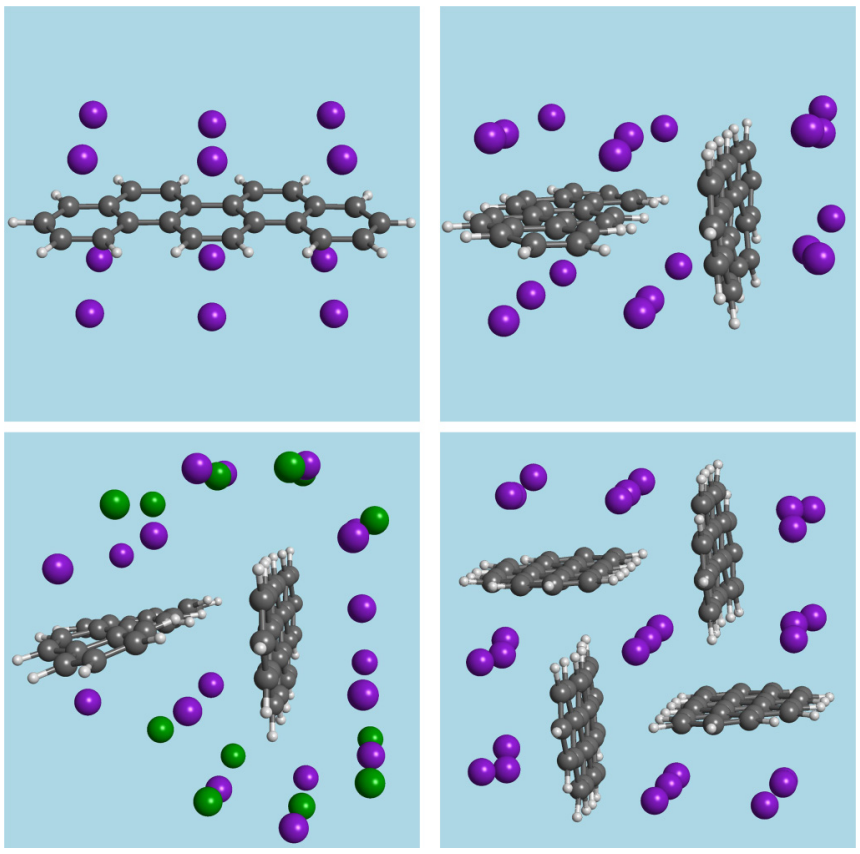

FIG. 1. (Color online) Potassium-picene clusters used in the present work. They are based in the probable crystalline structure of $\mathrm{K}_{3}$ picene and have been fully optimized at the DFT level using the B3LYP density functional. Numbering the figure panels from left to right and from top to bottom, the third one includes chlorine atoms to adjust charge transference from potassium atoms to picene molecules.

electrons of doped picene. It allows recovering the main results obtained for small clusters. In addition, solutions for larger systems give further support to our explanation of metallic charge transport. The work ends with a few final concluding remarks (Sec. V).

\section{COMPUTATIONAL PROCEDURES}

Potassium-doped picene clusters have been constructed based on the probable crystalline structure of $\mathrm{K}_{3}$ picene [18]. The edge-to-face arrangement of picene molecules is similar that found in pristine picene, while the positions of $\mathrm{K}$ atoms are not precisely known although all studies agree in having intralayer potassium that is more or less regularly distributed between aromatic molecules. In our study, we choose the most regular possible environments for picene molecules always surrounding them by $12 \mathrm{~K}$ atoms (see Fig. 1). The price we pay for this choice is a system that is too rich in potassium according to nominal stoichiometry. Nevertheless, we have found a procedure that works fine and correctly simulates the electronic structure of $\mathrm{K}_{3}$ picene. First, the neutral cluster is fully optimized at a DFT level. Secondly, the number of electrons in the cluster is reduced to make charge distribution as close as possible to that corresponding to the bulk material. In this way, three electrons are allowed to flow to picene molecules while the rest of the $\mathrm{K}$ valence electrons are assumed to be transferred to more distant molecules (that is, picene molecules that are not included in the cluster). Actually, charge transference from $4 s \mathrm{~K}$ orbital to LUMO and LUMO+1 or $\mathrm{LUMO}+2$ of picene is a bit more subtle since the third electron is not completely transferred to picene molecules. That means that K keeps part of its valence electron. After some trial and error we have found that about one-half of an electron remains in the groups formed by three almost aligned potassium atoms sitting between four picene molecules. Consequently, picene molecules gain only two and a half electrons and the total number of electrons of a cluster should be carefully modulated to be consistent with this situation. Prior to present detailed results, we would like to motivate the reader showing precisely the charge distribution on $\mathrm{K}$ orbitals predicted by our approach not only for the relatively small clusters that can be studied with high numerical precision but also for larger clusters that have been studied at a lower demanding computational cost (see Fig. 7 at the end of Sec. III).

In one case we have added chlorine atoms to the cluster to avoid changing the total number of electrons of the system after geometrical optimization. One electron is transferred from potassium atoms to each chlorine atom that is added to the cluster. Therefore, a $\mathrm{Cl}_{12} \mathrm{~K}_{18}$ picene 2 cluster has six valence electrons ready to populate picene LUMOs. However, DFT results show that two of these electrons remain on $\mathrm{K}$ atoms again demonstrating that the electron transfer from $\mathrm{K}$ to picene is not complete. Following a similar reasoning as used above for binary clusters, a complete charge consistency would imply some reduction of the number of chlorine atoms. This implies further geometry optimization and still lower symmetry around picene molecules; that is, strong reasons for not insisting along this line.

The electronic structure of clusters has been obtained using the US GAMESS $[19,20]$ program, which is a complete quantum chemistry package that allows both DFT calculations and precise configuration interaction $(\mathrm{CI})$ multireference calculations. When performing mean-field calculations, exchange and correlation are approximately included by the B3LYP functional [21-23]. This is a hybrid generalized gradient approximation functional combining five functionals, in particular, Hartree-Fock (HF) exchange. A large valence triple zeta plus polarization on all atoms (6-311G**) Gaussian basis has been chosen for H, C, and K atoms [24,25]. All graphical results have been obtained using the WXMACMOLPLT package [26].

\section{QUANTUM CHEMISTRY RESULTS}

\section{A. Picene molecule in a potassium environment}

This is the smallest K-picene system we have studied and the only one allowing state-of-the-art CI analysis. Based on the results of this case, we suggest that multireference calculations can be partially mimic at the DFT level increasing the spin multiplicity of the electronic state. In this way electrons can visit molecular orbitals that are not occupied in the singlet state. Sometimes even the total electronic energy decreases with increasing spin multiplicity. We do not give any significance to this fact since most probably a CI analysis would change the conclusions about the correct spin of the ground state.

To learn about the effect of $\mathrm{K}$ intercalation in picene crystal, one picene molecule is surrounded by $12 \mathrm{~K}$ atoms as happens in the crystalline structure. The positions of $\mathrm{K}$ atoms are forced to conserve the original $\mathrm{C}_{2 v}$ symmetry of the isolated polycyclic aromatic molecule just to make both computation and analysis 
easier. The precise geometry is obtained by minimization of the B3LYP total energy of the neutral cluster (the resulting cluster is represented in the upper left panel of Fig. 1). The final geometry does not differ appreciably from the crystalline one [18]. In fact, the mean distance of potassium atoms to the plane of the picene molecule is $2.91 \AA$, being deviations from this value less than $0.3 \AA$. Similar values hold for the other clusters shown in Fig. 1 [27]. After geometry optimization of the neutral cluster, nine electrons are removed from the cluster limiting to three the maximum number of electrons that potassium can transfer to the picene molecule. As has been said before, electrons removed from the cluster belong to neighboring cells that are not explicitly included in the study. Geometry is held fixed as a further optimization would artificially increase K-picene distances.

Our B3LYP calculations for the $\mathrm{K}_{12}$ picene $^{9+}$ cation indicate that while two electrons occupy the LUMO of isolated picene molecule, the third one remains in a symmetric combination of $4 s \mathrm{~K}$ orbitals (more precisely, the bonding combination of all twelve $4 s$ orbitals with almost equal coefficients that is shown in the upper right panel of Fig. 3 for the somewhat less charged $\mathrm{K}_{12}$ picene $^{8+}$ cation). Nevertheless, picene LUMO+1 and LUMO+2 are close in energy suggesting that they will play an important role if the one-configuration limitation is avoided. In fact, some tweaking of minor computational details such as geometry optimization or not of the ion, or keeping or not the symmetry group shows that the third valence electron can either occupy $\mathrm{K}$ orbitals or flow to some combination of picene $\mathrm{LUMO}+1$ and $\mathrm{LUMO}+2$. Consequently, a robust description of the electronic state requires at least a twoconfiguration wave function participated in by both $\mathrm{K}$ and picene orbitals. Here, DFT analysis was followed by a precise MCSCF (multiconfigurational self-consistent field) wavefunction study in which 16 orbitals describing the three valence electrons [28] were allowed to mix. That means 468 (480) determinants having space symmetry $A_{1}\left(B_{2}\right)$ for $S_{\mathrm{z}}=\frac{1}{2}$. Next, we will discuss the results obtained for the more interesting $B_{2}$ symmetry although the lowest energy obtained for this symmetry is somewhat higher $(0.31 \mathrm{eV})$ than that obtained for the $A_{1}$ wave function. We choose $B_{2}$ solution
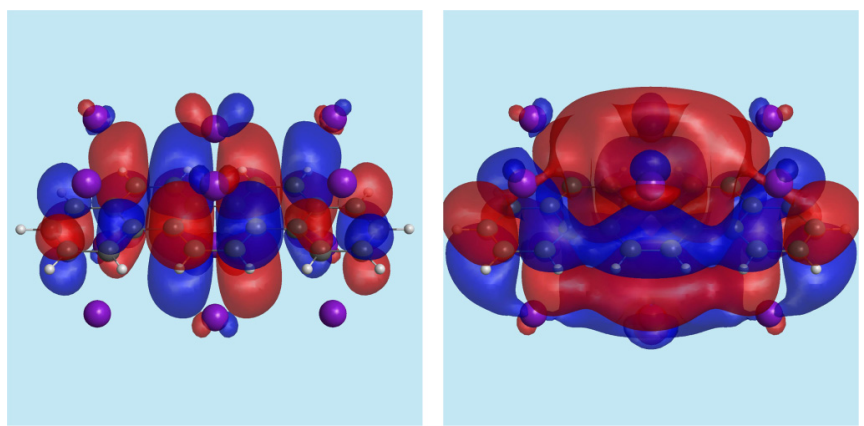

FIG. 2. (Color online) Most relevant natural orbitals obtained for the $\mathrm{K}_{12}$ picene $^{9+}$ cation, that is, a cluster populated by 362 core electrons and three valence electrons [28]. Orbital occupations are 1.941 (left orbital) and 0.992 (right orbital). Higher-energy orbitals show tiny occupations. The connection between electronic occupation of crystalline $\mathrm{K}_{3}$ picene and the number of electrons in clusters is discussed within the text (see Sec. III A). for symmetry reasons since $A_{1} \mathrm{~K}$ orbitals do not couple to $\pi$ electrons. The occupation of an $A_{1}$ molecular orbital (upper right panel of Fig. 3) would lead to a much larger charge on potassium atoms, i.e., to an almost pure $4 s \mathrm{~K}$ band showing half filling. Maybe this could provide an alternative explanation for metallic conductivity but we are strongly inclined to think that an ionic scenario showing partial charge transfers from $\mathrm{K}$ to picene is the most adequate.

Figure 2 shows the natural orbitals that describe the three valence electrons originating from $\mathrm{K}$ intercalation. The first one (left panel) is very similar to the LUMO of the isolated picene molecule and its occupation is 1.941. It is antisymmetric with respect to a plane cutting the picene molecule (and whole cluster) in two identical halves. On the other hand, the second one (right panel) is a mixture of picene LUMO+1 and LUMO+2 (both of $B_{2}$ symmetry) and $4 s$ potassium orbitals. It is symmetric relative to the above-mentioned plane and its occupation is 0.992 . Its hybrid character indicates that the third electron does not flow completely to picene orbitals but remains partially on $\mathrm{K}$ atoms. Since good quality $\mathrm{CI}$ computation is not possible for larger clusters, we will explore
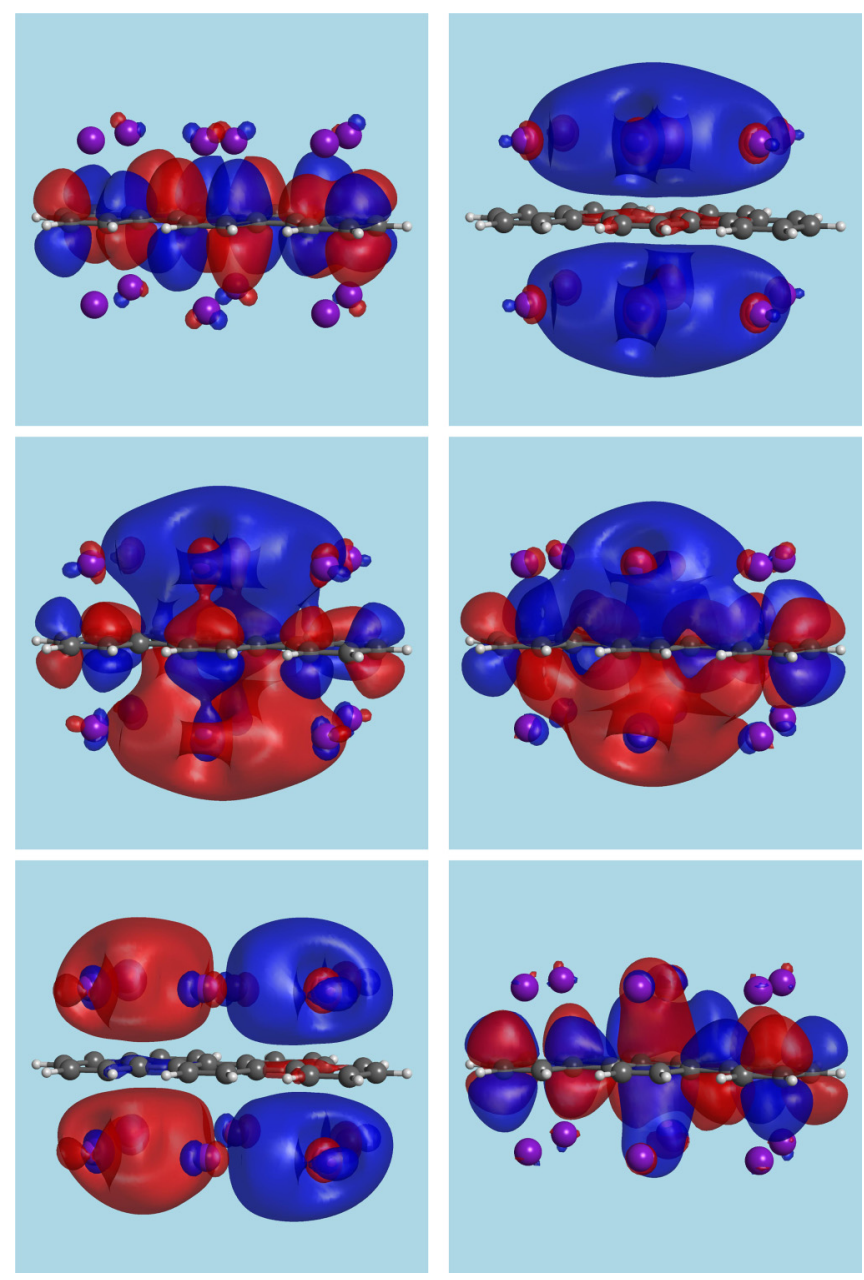

FIG. 3. (Color online) Molecular orbitals corresponding to valence electrons as obtained for the ground state (a spin triplet) of the $\mathrm{K}_{12}$ picene $^{8+}$ cluster. Following the numeration scheme stated in the caption of Fig. 1, the first orbital is doubly occupied, the next two are singly occupied, and the last three are empty. 
TABLE I. Energy of the lower spin excitations of doped and undoped picene clusters. The spin degeneracy of initial and final states is given in parentheses.

\begin{tabular}{|c|c|c|c|c|c|}
\hline System & First & Second & Third & Fourth & Fifth \\
\hline Picene & $2.23 \mathrm{eV}(1 \rightarrow 3)$ & $4.80 \mathrm{eV}(1 \rightarrow 5)$ & & & \\
\hline Picene $_{2}$ & $2.21 \mathrm{eV}(1 \rightarrow 3)$ & $4.48 \mathrm{eV}(1 \rightarrow 5)$ & & & \\
\hline Picene $_{4}$ & $2.15 \mathrm{eV}(1 \rightarrow 3)$ & $4.31 \mathrm{eV}(1 \rightarrow 5)$ & & & \\
\hline $\mathrm{K}_{12}$ picene $^{8+}$ & $0.082 \mathrm{eV}(3 \rightarrow 1)$ & $0.179 \mathrm{eV}(3 \rightarrow 5)$ & $3.23 \mathrm{eV}(3 \rightarrow 7)$ & & \\
\hline $\mathrm{K}_{18}$ picene $_{2}{ }^{10+}$ & $0.197 \mathrm{eV}(3 \rightarrow 5)$ & $0.311 \mathrm{eV}(3 \rightarrow 1)$ & $0.726 \mathrm{eV}(3 \rightarrow 7)$ & $0.671 \mathrm{eV}(3 \rightarrow 9)$ & $3.83 \mathrm{eV}(3 \rightarrow 11)$ \\
\hline $\mathrm{K}_{27}$ picene $_{4}{ }^{13+}$ & $0.043 \mathrm{eV}(7 \rightarrow 5)$ & $0.301 \mathrm{eV}(7 \rightarrow 3)$ & $0.358 \mathrm{eV}(7 \rightarrow 9)$ & $0.627 \mathrm{eV}(7 \rightarrow 11)$ & $0.685 \mathrm{eV}(7 \rightarrow 1)$ \\
\hline
\end{tabular}

now an easier way to partially recover these results. First, we argue that some additional electrons should be included in the cluster because our MCSCF calculation proves that only about two and a half electrons are transferred to the picene molecule, and consequently, about one half electron remains on every group formed by three $\mathrm{K}$ atoms. Therefore, the total count of valence electrons is $2.5+4 \times 0.5=4.5$ in this case, that is, one and a half more electrons than considered in the previous multiconfigurational analysis. As subsequent calculations at a DFT level evidence, the addition of just one extra valence electron to the system suffices to end with a nearly consistent charge both on picene and $\mathrm{K}$ groups. Let us remark here that although this is our preferred choice, the main conclusions are the same for three, four, or five valence electrons. Secondly, larger spin degeneracies that partially populate higher molecular orbitals are considered. In this case, a spin triplet provides the best total electronic energy from a single-determinantal point of view. The calculated MOs are shown in Fig. 3. The first one is again picene LUMO, the second one is mainly $4 s \mathrm{~K}$, while the third (singly occupied) and fourth (empty) show the characteristics we are looking for in our study. Both are antisymmetric relative to the plane containing the picene molecule and both show an important hybridization between picene and potassium orbitals. As a matter of fact, the empty fourth MO is very similar to the occupied one obtained in the more precise MCSCF description (see Fig. 2). This orbital becomes singly occupied for a spin quintuplet but the energy price paid for the excitation of one of the electrons on the LUMO is important (Table I gives the details). A rough electron counting based exclusively on thepictures gives 2.5 electrons on picene and 1.5 electrons on $\mathrm{K}$ atoms, which is satisfactorily consistent with our assumption for the distribution of the valence charge.

\section{B. $\mathrm{K}_{18}$ picene ${ }_{2}^{10+}$ cation}

In this section, the results for two picene molecules in an edge-to-face conformation surrounded by 18 potassium atoms are presented (upper right panel of Fig. 1 shows the cluster). The geometry of the cluster has been optimized using DFT for the neutral system. Afterward, the electronic structure of a cluster with less electrons in the outer shell has been studied. Based on our experience, we assume that half an electron remains on every $\mathrm{K}$ group formed by three atoms. That means a total number of three electrons on $\mathrm{K}$ atoms that added to the five electrons transferred to picene molecules gives a total number of eight valence electrons in the cluster. Therefore, ten electrons are removed from the neutral cluster to end with a $10+$ cation. Several spin multiplets have been obtained for this system, being a spin triplet its ground state. Molecular orbital energies corresponding to valence electrons are about $3 \mathrm{eV}$ above the highest core electron molecular orbital energy. Figure 4 shows the first nine molecular orbitals (MOs) in the valence band region. The first and the third correspond to the LUMO of neutral picene, while the rest show an important $\mathrm{K}$-picene hybridization. It is easy to see that the number of electrons on $\mathrm{K}$ atoms is fully consistent with our initial assumption of just three electrons on $\mathrm{K}$ atoms. Table I gives a detailed account of the low-energy spin excitations of this system. The role played by electronic excitations will be discussed later.

\section{C. $\mathrm{Cl}_{12} \mathrm{~K}_{18}$ picene 2 neutral cluster}

A graphical representation of the MOs corresponding to this ternary cluster is shown in Fig. 5. The number of valence electrons in the cluster is easily obtained in this case. It is simply the number of $\mathrm{K}$ atoms minus the number

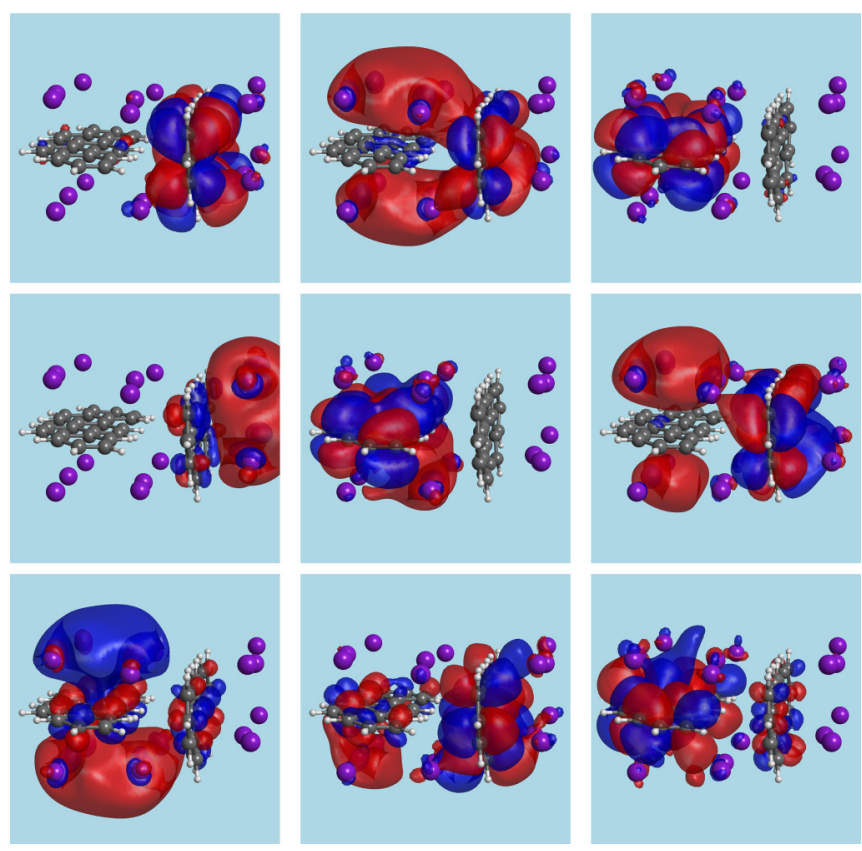

FIG. 4. (Color online) Molecular orbitals of valence electrons obtained for the ground state (a spin triplet) of the $\mathrm{K}_{18}$ picene ${ }_{2}{ }^{10+}$ cluster. Following the numeration scheme stated in the caption of Fig. 1, the first three orbitals are doubly occupied, the next two are singly occupied, and the rest are empty. 

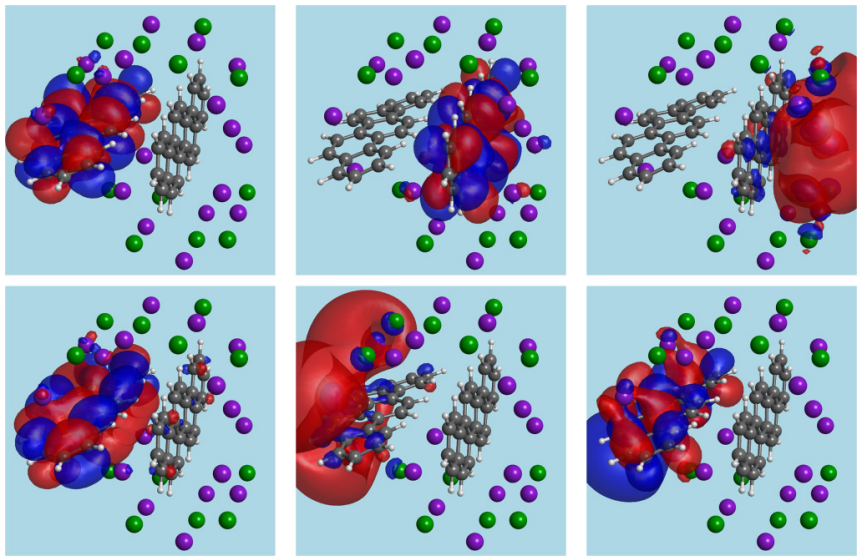

FIG. 5. (Color online) Higher molecular orbitals (valence electrons) of a $\mathrm{Cl}_{12} \mathrm{~K}_{18}$ picene 2 neutral cluster designed to simulate the electronic occupation of crystalline $\mathrm{K}_{3}$ picene. Four electrons occupy MOs based on the LUMOs of isolated picene molecules, while the last two electrons go to a symmetric combination of potassium $4 s$ orbitals (shown in the top right panel).

of chlorine atoms (six for the present composition). As happened in simpler binary clusters, valence electrons do not flow completely to unoccupied molecular orbitals of picene molecules. In fact, four electrons do populate picene LUMOs but the next two go to a symmetric combination of $4 s \mathrm{~K}$ orbitals. Therefore, the number of chlorine atoms should be reduced to get the correct K-picene hybridization. Since this calculation would require a completely new cluster and the subsequent very tedious geometrical optimization of the structure and the effects are better described by binary clusters, we have not followed this line of research any longer.

\section{Four picene molecules modeling $K_{3}$ picene}

The DFT optimized geometry obtained for this cluster is shown in Fig. 1 (bottom right panel). An extra $C_{2 h}$ symmetry group was assumed that is not shared by the crystalline structure. It makes geometry optimization faster and allows an easier analysis of its electronic structure. Following previous methodology, 14 valence electrons have been considered in this case after geometrical optimization of the cluster anion (just one extra electron to close shells). Ten of them are on picene molecules and the rest on potassium atoms. Figure 6 shows the relevant MOs corresponding to a spin heptuplet which gives the lowest B3LYP DFT energy (see Table I for details). There are four electrons occupying symmetric combinations of $4 s$ orbitals on potassium groups and two electrons that are mostly located on picene described by a particular combination of picene LUMO +1 and LUMO +2 and some $4 s \mathrm{~K}$ orbitals. The last two electrons together with eight electrons on picene LUMOs give a total number of ten valence electrons on picene molecules. Additional orbitals that are very close in energy are also shown in the figure.

When the results for the single-determinantal electronic structure of this system are analyzed together with excitation energies given in Table I and the experience gained by the $\mathrm{CI}$ analysis of the simplest cluster, the main message of our work is obtained. Namely, after doping picene molecules with
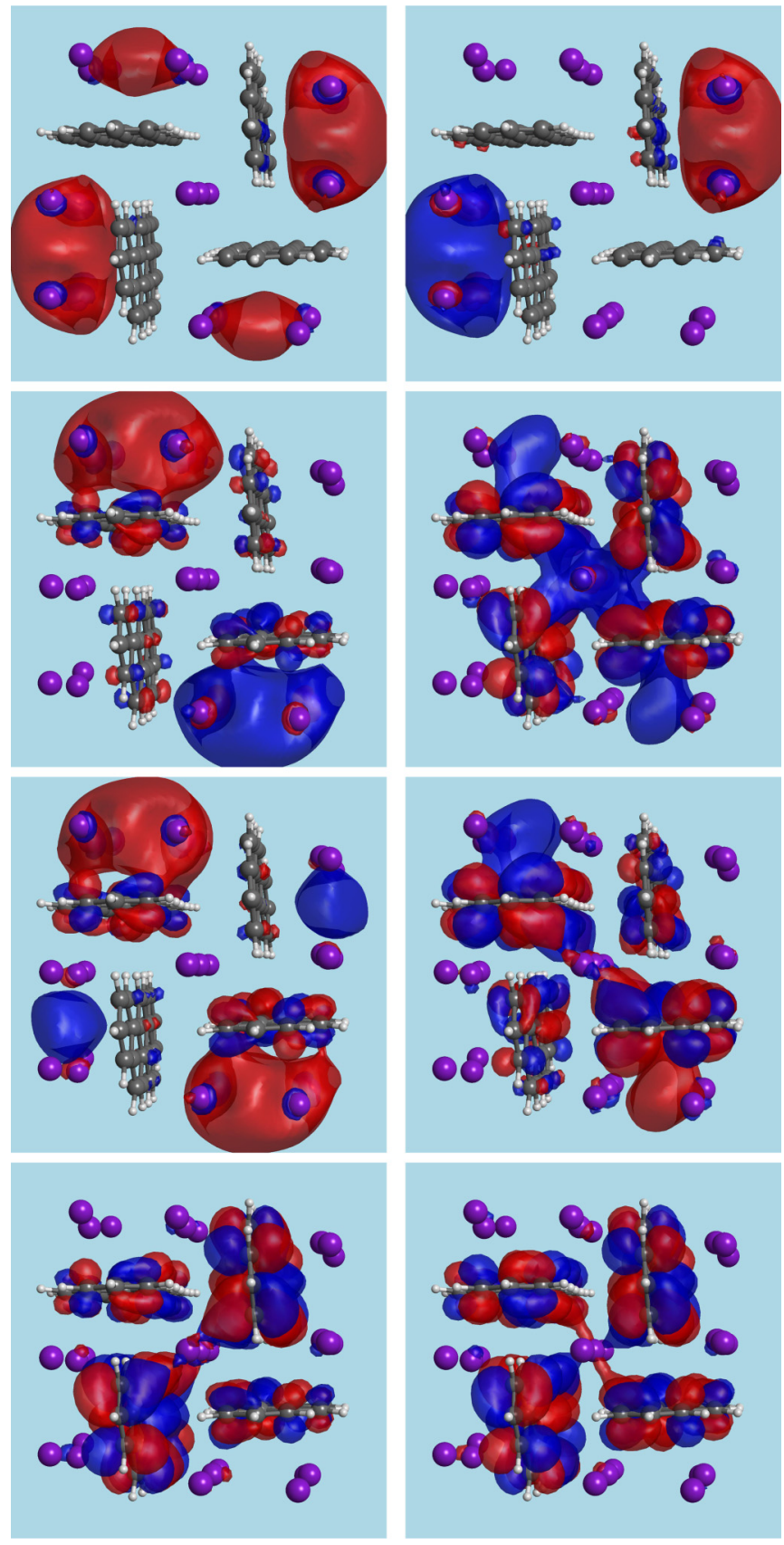

FIG. 6. (Color online) Most relevant molecular orbitals of the ground state (a spin heptuplet) obtained for the $\mathrm{K}_{27}$ picene $_{4}{ }^{13+}$ cluster. The eight deeper valence electrons going to combinations of picene LUMOs are not shown. The six remaining valence electrons singly occupy the first six orbitals shown in the figure (following again the numbering convention stated in the caption of Fig. 1). The last two orbitals completing the LUMO+1-LUMO+2 shell are empty.

two electrons their reduced electronegativity is not enough to gain the extra electron offered by potassium atoms. This last electron should be described as belonging simultaneously to $\mathrm{PAH}$ molecules and alkali atoms. However hopping between $\mathrm{LUMO}+1$ or LUMO +2 and $4 s \mathrm{~K}$ orbitals is very small mainly due to the rich structure of picene molecular orbitals that prevents the existence of a naive chemical bond. Therefore, we prefer to say that the electron can hop between apparently 
separated $\mathrm{K}$ groups just because the intermediate picene molecule is able to transfer the electron from one group to the other [17]. It can be said alternatively using a band theory language that picene $\mathrm{LUMO}+1$ and LUMO +2 become somewhat hybridized with the $\mathrm{K} 4 s$ state giving rise to a very narrow band that is partially occupied (one-fourth if two bands are entangled but one-sixth if three bands are coupled). In fact, an independent estimation of the hopping energy provides a value below one-tenth of an $\mathrm{eV}$ resulting in an estimated bandwidth of a few tenths of eV. Since it is well known that only half-filled Hubbard models on bipartite lattices lead to an unquestionable insulator when correlation effects dominate, our results strongly suggest that the main reason for the metallic behavior of $\mathrm{K}_{3}$ picene compounds is the small occupation of the hybridized band. We explore in the next section the behavior of the simplest interaction model describing this material. Good approximate solutions can be obtained for larger systems. Results enrich the image obtained for small all-electron systems and give further support for a metallic behavior of K-intercalated picene even in the presence of very strong electronic correlations.

Let us finish this section by commenting on Table I. The table shows the spin excitation energies for the three K-picene clusters shown in Fig. 1 plus the clusters produced removing $\mathrm{K}$ atoms. We wished to obtain additional electronic excitations for fixed spin multiplicities but this is a difficult effort in computational chemistry. Nevertheless, an increased wealth of excitations are obtained for the interaction model in the next section. The results for the undoped clusters undoubtedly indicate the existence of a gap of about $2.2 \mathrm{eV}$ in the electronic spectrum of picene molecules. Spin excitations are produced promoting one paired core electron to an unoccupied level with a large energy cost. Flipping an additional electron to produce a spin quintuplet implies two electrons in excited states and more than twice the gap energy. On the other hand, spin excitation energies are much smaller for the doped clusters pointing therefore to a dense spectrum of many-body states. Also notice that excitation energy becomes above $3 \mathrm{eV}$ when an electron on a picene LUMO needs to be promoted to a higher energy level to form the spin multiplet. In short, we attribute the existence of small energy electronic excitations of doped clusters to the richness of $\mathrm{LUMO}+1-\mathrm{LUMO}+2-4 s(\mathrm{~K})$ configurations participating in accurate many-body wave functions.

\section{E. $K$ charge in a large $K_{3}$ picene cluster}

A large $\mathrm{K}_{3}^{3+}\left(\mathrm{K}_{3} \text { picene }\right)_{12}$ cluster taken from our crystalline structure (Ref. [10]) has been solved using the B3LYP functional and a PC1 Gaussian basis [29]. The cluster and crystal are isoelectronic in this case. The total number of valence electrons is 36 . These valence electrons populate picene molecular orbitals that are unoccupied in the pristine material and K orbitals. Figure 7 shows the charge distribution associated to $\mathrm{K}$ atoms. At the corners it is similar to the one found on smaller clusters for external $\mathrm{K}_{6}$ groups while it follows the one found for the confined $K_{3}$ group of $\mathrm{K}_{27}$ picene $_{4}{ }^{13+}$ cation for all potassium groups surrounded by four picene molecules. The charge density isosurface has been plotted at a small value of $3 \times 10^{-5} e \AA^{-3}$ to show that an
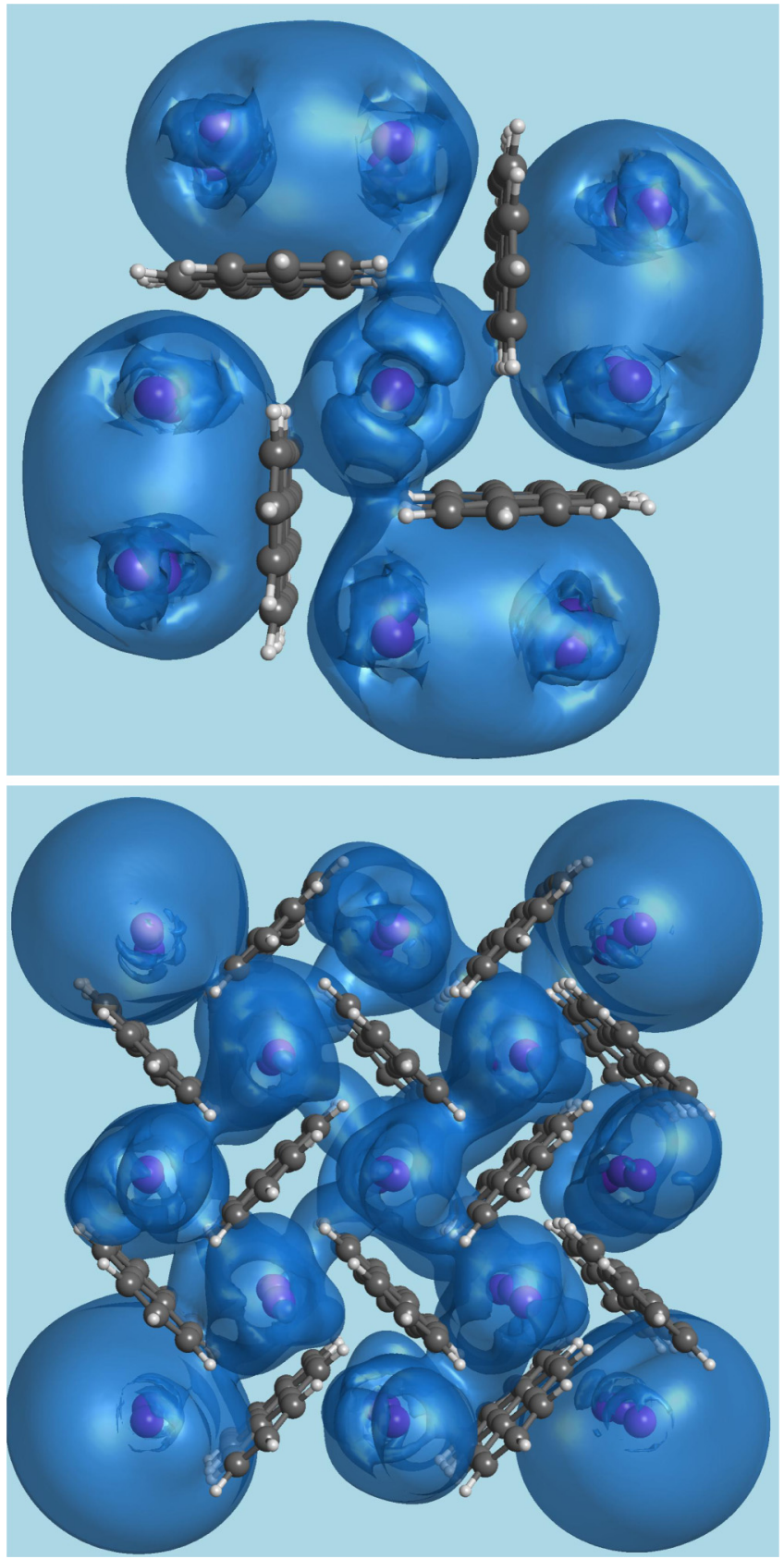

FIG. 7. (Color online) Charge density corresponding to valence electrons projected onto $\mathrm{K}$ orbitals. The distribution in the cluster studied in Sec. III D (upper panel) is compared with the distribution of a much larger cluster (lower panel).

overlap between $\mathrm{K}$ and picene orbitals should exist although its value will be presumably tiny.

\section{MANY-BODY MODEL HAMILTONIAN}

\section{A. Charge gap}

A simple way to infer values for the energies due to interaction among electrons in a cluster is changing the total number of electrons in it. Table II gives the values obtained for our clusters not only when $\mathrm{K}$ is present but also when potassium is completely removed from the system. The 
TABLE II. Charge gap calculated for both doped and undoped picene clusters using expression $E_{N+1}+E_{N-1}-2 E_{N}$ where $E_{N}$ is the total DFT energy of the reference system ( $N$ electrons).

\begin{tabular}{lccc}
\hline \hline Doped cluster & Gap energy & Undoped cluster & Gap energy \\
\hline $\mathrm{K}_{12}$ picene $^{8+}$ & $2.72 \mathrm{eV}$ & Picene & $6.10 \mathrm{eV}$ \\
$\mathrm{K}_{18}$ picene $_{2}{ }^{10+}$ & $2.37 \mathrm{eV}$ & Picene $_{2}$ & $5.34 \mathrm{eV}$ \\
$\mathrm{K}_{27}$ picene $_{4}{ }^{13+}$ & $1.83 \mathrm{eV}$ & Picene $_{4}$ & $4.86 \mathrm{eV}$ \\
\hline \hline
\end{tabular}

importance of size effects can be correctly estimated by the comparison of the values obtained in doped systems with the ones obtained for pure systems. Actually, we calculate the charge gap $E_{N+1}+E_{N-1}-2 E_{N}$ for a cluster with $N$ electrons in the reference electronic state. It measures the insulating character of the system. It is obviously always finite for clusters but can go to zero in infinite systems. Values for undoped clusters (fourth column) are much larger than for K-doped systems (second column) and both show a clear diminution with size. We know that values for the last column should tend to the semiconducting gap of picene (about $2.2 \mathrm{eV}$ ) but it is impossible to say what will happen with the charge gap for crystalline $\mathrm{K}_{3}$ picene although an eventual size scaling towards zero could occur. Here it is a good place to remember some works emphasizing that quarter-filled bands can give metallic conduction in spite of showing a finite charge gap [30-32]. Moreover, small energy spin excitations such as those included in Table I for doped clusters point to metallic behavior not only because they could approach zero for larger systems but also because they imply charge displacement [33].

\section{B. Two-band model}

Here we propose a minimalist interaction model Hamiltonian for $\mathrm{K}_{3}$ picene crystal. It is based on the main result of our quantum chemistry calculations that show an important hybridization between picene and potassium orbitals in spite of the very small direct coupling. A straightforward calculation of the Hubbard $U$ (intrasite Coulomb repulsion) gives between 3 and $4 \mathrm{eV}$ both on picene and on a group of three aligned $\mathrm{K}$ atoms. The precise value depends on details such as occupation, geometry, and others. Coulomb repulsion for separate electrons can be estimated from the Coulomb law. The last ingredient of the model is hopping from picene LUMO+1 or LUMO +2 to bonding $4 s$ combinations. It is necessarily small since unoccupied $\pi$ states of picene show alternating positive and negative lobes while $4 s$ combinations show a very mild spatial dependence. In order to get a concrete value for the hopping we have used a simple multiconfigurational calculation based on the two orbitals that show the main coupling in the simplest system (the $\mathrm{K}_{12}$ picene $^{9+}$ cluster presented in Sec. III A).

Parameters of the interacting model are obtained from a series of simple CI calculations of the $\mathrm{K}_{12}$ picene cluster shown at the upper left corner of Fig. 1. Many-body states are calculated for electronic populations that go from two to six valence electrons. Two electrons occupy an $A_{2}$ molecular orbital (very similar to the first one of Fig. 2) while the rest populate two $\mathrm{B}_{2}$ molecular orbitals, that is, orbitals showing the symmetry of the second natural orbital of Fig. 2 but localized on picene or $\mathrm{K}$ atoms at the mean-field level. The energy of the state with two electrons on the slightly modified picene LUMO ( $A_{2}$ molecular orbital) is considered as the reference. Both CI energies and wave functions are taken into account to fit the parameters of an interacting model now including only two states. One corresponds to picene and the second to K. We start fitting the case of one extra electron which is described by the following Hamiltonian matrix:

$$
\mathbf{H}_{1 e}=\left(\begin{array}{cc}
\epsilon_{1} & t \\
t & \epsilon_{2}
\end{array}\right),
$$

where $\epsilon_{i}$ are the state levels and $t$ the hopping between them. The following values are obtained: $\epsilon_{1}=-24.00 \mathrm{eV}$, $\epsilon_{2}=-23.95 \mathrm{eV}$, and $t=-0.048 \mathrm{eV}$. Eigenvector amplitudes are $(0.846252,0.532783)$ and $(-0.532783,0.846252)$ for both $a b$ initio and model calculations.

The Hamiltonian matrix describing two extra valence electrons is

$$
\begin{aligned}
& \mathbf{H}_{2 e} \\
& =\left(\begin{array}{cccc}
2 \epsilon_{1}+U_{1} & 0 & t & t \\
0 & 2 \epsilon_{2}+U_{2} & t & t \\
t & t & \epsilon_{1}+\epsilon_{2}+V & 0 \\
t & t & 0 & \epsilon_{1}+\epsilon_{2}+V
\end{array}\right),
\end{aligned}
$$

where $U_{i}$ are on-site Coulomb repulsion energies while $V$ gives the Coulomb repulsion energy between picene-centered and K-centered molecular orbitals. The triplet state $(0,0,1,-$ 1) allows the determination of $V$. We get $V=2.99 \mathrm{eV}$. The on-site Coulomb parameters are obtained diagonalizing the Hamiltonian matrix describing three extra valence electrons:

$$
\mathbf{H}_{3 e}=\left(\begin{array}{cc}
2 \epsilon_{1}+\epsilon_{2}+U_{1}+2 V & t \\
t & \epsilon_{1}+2 \epsilon_{2}+U_{2}+2 V
\end{array}\right) .
$$

Values of $U_{1}=3.31 \mathrm{eV}$ and $U_{2}=4.81 \mathrm{eV}$ are obtained. Eigenvector amplitudes are $(0.994478,-0.104948)$ and $(0.104948,0.994478)$ for both ab initio and model calculations. Finally, the case of four extra electrons filling completely both $B_{2}$ molecular orbitals is described by

$$
\mathbf{H}_{4 e}=\left(2 \epsilon_{1}+2 \epsilon_{2}+U_{1}+U_{2}+4 V\right) .
$$

Singlet states for two electrons are obtained by diagonalization of $\mathbf{H}_{2 e}$. They are given in Table III compiling all $a b$ initio and model energies. Agreement is good for the three singlets and also for the completely filled system.

Finally, a hopping of $-0.05 \mathrm{eV}$ correctly fits $a b$ initio results. On the other hand, alternative values for the Coulomb integrals are obtained as a by-product. Notice that $U / t \sim$ 60-80, which implies a strongly correlated electronic system that would lead to an insulating behavior in any bipartite lattice. As a further simplifying detail, we will study an isolated layer of $\mathrm{K}_{3}$ picene ignoring possible three-dimensional effects. Figure 8 shows a $6 \times 6$ cluster of the model in which both the perspective and the large size of atoms helps to imagine the relevant orbitals. The bidimensional array is formed by alternating picene and $\mathrm{K}$ sites in an underlying square lattice. Nevertheless, the model differs from a simple interaction model on the square lattice because the sign of the hopping energy should be carefully chosen to take into account both 
TABLE III. Energies of a two-state interacting model as a function of electron population. Energies of the two last columns refer to the empty state.

\begin{tabular}{lccc}
\hline \hline $\begin{array}{l}\text { Number of } \\
\text { extra electrons }\end{array}$ & $\begin{array}{c}\text { CI eigenenergy } \\
\text { (hartree) }\end{array}$ & $\begin{array}{c}\text { Ab initio } \\
\text { energy }(\mathrm{eV})\end{array}$ & $\begin{array}{c}\text { Model } \\
\text { energy }(\mathrm{eV})\end{array}$ \\
\hline 0 & -7988.5174 & 0 & 0 \\
1 & -7989.3958 & -24.03 & -24.03 \\
1 & -7989.3919 & -23.92 & -23.92 \\
2 & -7990.1656 & -44.98 & -44.98 \\
2 (triplet) & -7990.1650 & -44.96 & -44.96 \\
2 & -7990.1535 & -44.65 & -44.67 \\
2 & -7990.0973 & -43.12 & -43.10 \\
3 & -7990.8159 & -62.67 & -62.67 \\
3 & -7990.7581 & -61.10 & -61.10 \\
4 & -7991.2991 & -75.82 & -75.82 \\
\hline \hline
\end{tabular}

the $\pi$ character of PAH molecular orbitals and the particular staggering of picene molecules in the herringbone structure.

The model Hamiltonian contains a noninteracting part $\hat{H}_{0}$ and a term that incorporates the electron-electron interactions $\hat{H}_{I}$ :

$$
\hat{H}=\hat{H}_{0}+\hat{H}_{I}
$$

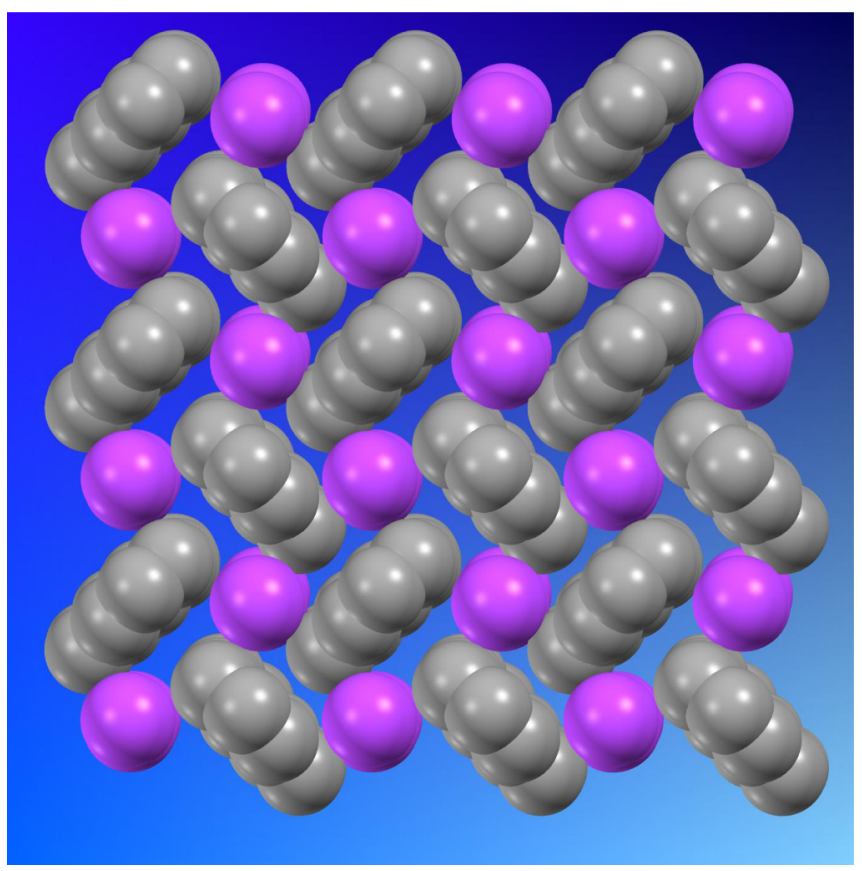

FIG. 8. (Color online) $6 \times 6$ cluster illustrating our interaction model study. The large size of atoms ( $80 \%$ of the Van der Waals radii) tries to simulate spatial extension of involved orbitals: LUMO+1 or LUMO +2 on picene molecules and bonding $4 s$ combinations on the $\mathrm{K}$ rows. We ignore direct hopping between picene orbitals owing to their symmetry. Consequently, hopping is only considered between $\mathrm{LUMO}+1 s($ or $\mathrm{LUMO}+2 s$ ) and their neighboring four rows of $\mathrm{K}$ atoms. In the same way, hopping of $\mathrm{K} 4 s$ combinations is limited to their four neighboring picene orbitals. Although all hopping energies are taken equal for simplicity, signs take into account the $\pi$ character of picene orbitals.
The noninteracting term is written as

$$
\hat{H}_{0}=\sum_{i \sigma} \epsilon_{i} \hat{c}_{i \sigma}^{\dagger} \hat{c}_{i \sigma}+\sum_{i j \sigma} t_{i j} \hat{c}_{i \sigma}^{\dagger} \hat{c}_{j \sigma}
$$

where the operator $\hat{c}_{i \sigma}^{\dagger}$ creates an electron at site $i$ with spin $\sigma, \epsilon_{i}$ is the energy of the orbital at site $i$, and $t_{i j}$ is the hopping between orbitals at sites $i$ and $j$, respectively.

The interacting part is given by

$$
\hat{H}_{I}=U \sum_{i} \hat{n}_{i \uparrow} \hat{n}_{i \downarrow}+\frac{1}{2} \sum_{i j ; i \neq j} V_{i j}\left(\hat{n}_{i}-1 / 2\right)\left(\hat{n}_{j}-1 / 2\right),
$$

where $U$ is the on-site Coulomb repulsion and $V_{i j}$ is the intersite Coulomb repulsion given by

$$
V_{i j}=U\left[1+\left(\frac{U}{e^{2} / d_{i j}}\right)^{2}\right]^{-1 / 2},
$$

being $d_{i j}$ the distance between $i$ and $j$ sites.

The electronic density operator for spin $\sigma$ at site $i$ is

$$
\hat{n}_{i \sigma}=\hat{c}_{i \sigma}^{\dagger} \hat{c}_{i \sigma},
$$

whereas the operator giving the total electron density at the same site is

$$
\hat{n}_{i}=\hat{n}_{i \uparrow}+\hat{n}_{i \downarrow} .
$$

Let us remind one that we use $\epsilon_{i}=0 \mathrm{eV}, t_{i j}= \pm 0.05 \mathrm{eV}$ for nearest neighbors and $U=4.0 \mathrm{eV}$ in an underlying square lattice of lattice constant equal to $3.5 \AA$ as a minimum model describing resonance between picene and $\mathrm{K}$ valence electrons. Sometimes we have used an ionic variation of this model ( $\epsilon_{i}=0$ on picene sites but $\epsilon_{i}=0.4 \mathrm{eV}$ on $\mathrm{K}$ sites) to check that qualitative results do not depend on the oversimplification of the proposed model.

The model Hamiltonian is solved using a development of the Lanczos method that follows quantum chemistry standards. A mean-field approach is used to obtain uncorrelated HF molecular orbitals. Afterward, the interaction model is rewritten in this new basis and CI employed. The crucial point is that selection of configurations is not based on the occupation of the initial wave function but generated from it by successive applications of the many-body Hamiltonian (keep in mind that the use of the Hamiltonian operator to increase the Hilbert space dimension constitutes the essence of the Lanczos method). New configurations are kept only when their participation in the ground state is relevant, that is, when its amplitude is above some carefully chosen threshold. If the threshold is too small, the number of configurations exceeds computational capabilities. On the other hand, if the threshold is too large only a few configurations are generated and correlation is poorly included. Typically, no more than several hundreds of thousands of configurations are used to describe the correlated system. However, the way they have been selected produces a final result showing very good quality. Although our method is applied to a model Hamiltonian and a purely numerical algorithm is used for the selection of configurations, we believe that it shares some similarities with a well documented set of wise criteria of CI selection used for $a b$ initio calculations [34]. Further details on the whole Lanczos procedure can be found in recent papers $[16,35]$. 


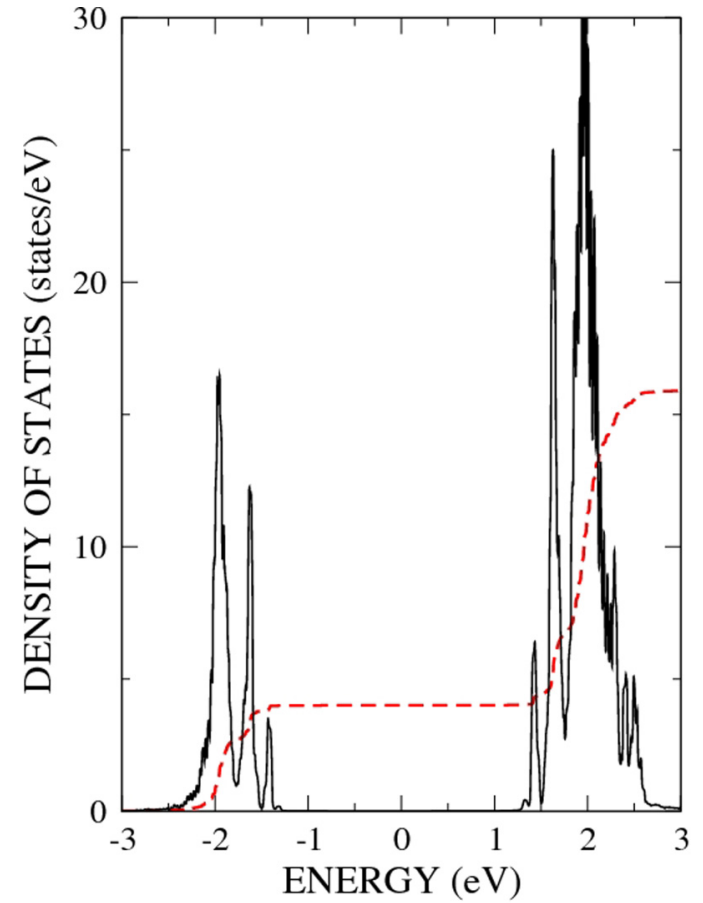

FIG. 9. (Color online) The density of states (DOS) of a $4 \times 4$ cluster with eight electrons described by the model Hamiltonian is shown in black. The lower band is fully occupied (four spin-up electrons and four spin-down electrons) while the upper one is empty. Integrated occupation per spin is given by the dashed red line (the numerical scale is valid for the integrated DOS).

\section{Some exact results for a small $4 \times 4$ cluster}

The density of states of a $4 \times 4$ cluster has been calculated within Lanczos formalism for the minimalist model Hamiltonian described in the precedent section. The simplest version with $\epsilon_{i}=0$ for all sites $i$ was chosen. The result is given in Fig. 9. It shows two Hubbard bands centered on $\pm \frac{1}{2} U$ and separated by an energy equal to the charge gap obtained from the data in Table IV. The lower band is completely filled with eight electrons (four of each spin) while the upper one is empty.

Table IV collects some exact results for the same $4 \times 4$ cluster using in this case both covalent and ionic flavors of the lattice model. Anion and cation energies together with the ground

TABLE IV. Exact results for the interaction model on a small $4 \times 4$ cluster. The second column corresponds to equal levels on all sites while the third column gives the values for the more general ionic version. A large number of digits is necessary to show the very small energy differences between the lowest many-body states.

\begin{tabular}{lcc}
\hline \hline State & $E_{\text {covalent }}(\mathrm{eV})$ & $E_{\text {ionic }}(\mathrm{eV})$ \\
\hline Ground state & -7.568838 & -7.534038 \\
First excited singlet & -7.568685 & -7.533984 \\
Second excited singlet & -7.568658 & -7.533972 \\
First triplet & -7.568823 & -7.534021 \\
First quintuplet & -7.568812 & -7.534003 \\
Anion GS & -6.275474 & -5.617117 \\
Cation GS & -6.258459 & -5.930108 \\
\hline \hline
\end{tabular}

state energy allow obtaining the charge gap. Values of 2.60 and $3.52 \mathrm{eV}$ result for covalent and ionic models, respectively. They overestimate values in Table II pointing to an excessively large $U$ value. On the other hand, excitation energies are much smaller than those given in Table I for all-electron clusters. This fact points again to an unrealistic dominance of interaction over hopping. Therefore, it should be inferred that the many-body physics contained in the quantum chemistry results is not completely reproduced by the simplest, model that needs at least an ad hoc modulation of the $U / t$ ratio. In any case, diminishing $U / t$ is always possible and will lead to a reinforcement of the signals pointing to a metallic behavior of the system.

\section{HOMO-HOMO correlation function}

Lanczos methods not only allow the calculation of the correlated many-body wave function but also obtaining dynamical properties such as response functions or correlation functions. After the selection of some convenient operator $\hat{O}$, it is applied to the ground state at $t=0$ and propagated up to time $t$ using the exact many-body Hamiltonian. After Fourier transforming the time variable, correlation is given by

$$
C(\omega)=-\frac{1}{\pi} \operatorname{Im}\left\langle\mathrm{GS}\left|\hat{O}^{\dagger}\left(\omega+E_{\mathrm{GS}}+i \epsilon-\hat{H}\right)^{-1} \hat{O}\right| \mathrm{GS}\right\rangle,
$$

where $|\mathrm{GS}\rangle$ is the many-body ground state and $E_{\mathrm{GS}}$ its energy. The propagator can be written in tridiagonal form using a Lanczos representation of Hilbert space (see Sec. II.A.2 of Dagotto's review [36]). Here, we will follow the time response of the system to the creation of an electron-hole pair to reveal low-energy excitations that does not change the total number of particles in the system. Usually, Lanczos formalism is used to get one-electron Green functions in which case one electron (or hole) is created at $t=0$ and its time evolution followed before destruction at time $t$. In these cases, time propagation occurs via many-body states containing one more (or one less) particle and the correlated density of states is obtained. Since we know that a large charge gap will separate empty from occupied states due to size effects, we prefer to infer information from two-particle electronic excitations using correlation functions. In fact, the time evolution of a pair excitation obtained by annihilation of one electron in an antibonding combination of HOMOs and creation in the bonding combination has been calculated, i.e.,

$$
\hat{O}=\hat{c}_{\text {homo1 }}^{\dagger} \hat{c}_{\text {homo2 }},
$$

where homo1 stands for the bonding linear combination of the two HOMOs that produces the preliminary mean-field calculation, whereas homo 2 stands for the antibonding linear combination of the same MOs.

Results corresponding to $4 \times 4,6 \times 6$ and $8 \times 8$ clusters are shown in Fig. 10. We have checked that mean-field HOMOs are strongly localized on distant lattice sites. Therefore, $C(\omega)$ can be seen as the time response to a large dipole perturbation. It starts displaying several peaks at small energies (some tenths of eV) that are followed by an incoherent part at larger energies (one or two eV). In this way, the existence of small energy electronic excitations involving charge displacement is proved for relatively large clusters. A more detailed exposition 


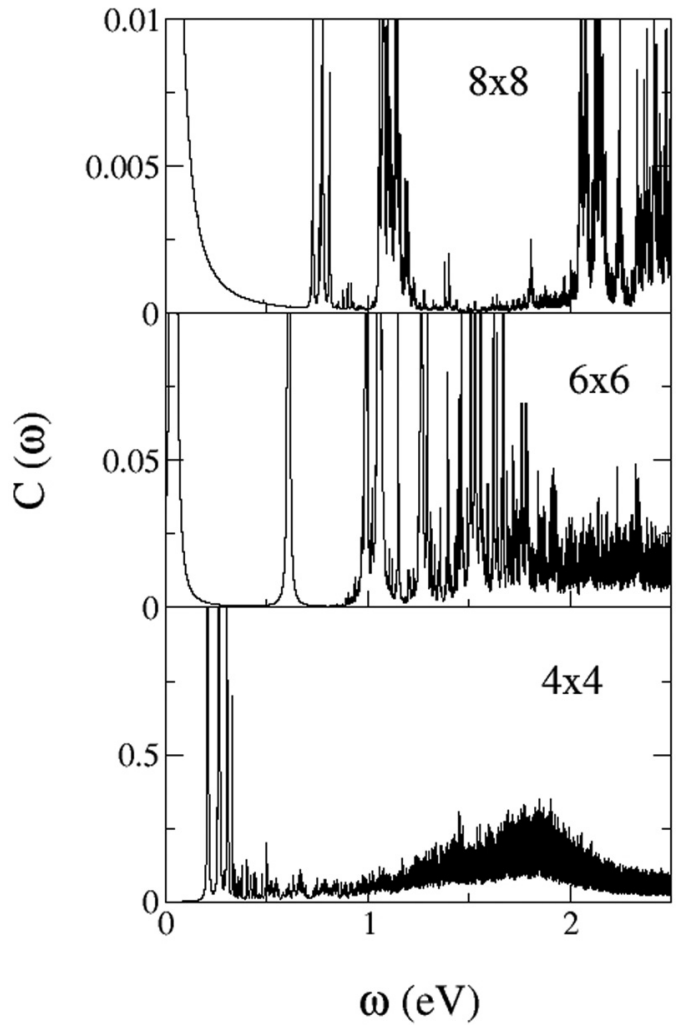

FIG. 10. HOMO-HOMO correlation function defined by Eqs. (7) and (8) as a function of the cluster size. The time evolution of an HOMO-HOMO electron-hole pair is followed being the pair formed by bonding and antibonding linear combinations of the two HOMOs obtained in the initial mean-field approximation of the correlated state.

of Lanczos methods and results is postponed for a future publication.

\section{CONCLUDING REMARKS}

We want to finish the presentation of our basically computational work with a few remarks based on the more robust results. (i) Doping of picene with potassium completely populates the LUMO of picene producing a band that is inert from the point of view of electronic transport. (ii) The third electron of the $\mathrm{K}_{3}$ group is not transferred to picene LUMO+1 or LUMO+2 but shared among PAH molecules and $\mathrm{K}$ atoms in spite of the very small hopping between the corresponding orbitals. (iii) Since hopping is small but interaction is large, the correct description of the electronic structure requires a many-body formulation. (iv) Both quantum chemistry results for small all-electron clusters and results obtained from a minimalist interaction model coincide in getting small energy electronic excitations that imply charge displacement. (v) These results open the way to explain metallic behavior in spite of the strong interaction shown by electrons at the Fermi level.

A comparison of our results with previous theoretical contributions allows one to find some similarities with the works of Kim et al. that assign metallic behavior to complex Fermi surfaces formed by several bands [11,37] but also important disagreement with conclusions derived by other authors based on the large gap shown by the spectral function of the strongly correlated electronic system (see Fig. 3 of Ref. [14]). Notice that our interaction model is similar to theirs (although we are coupling $\mathrm{K}$ and picene bands and our $U$ value is sensibly larger), and therefore, our quarter-filled two-band model produces a spectral function that resembles the one shown at the top of the mentioned figure (Fig. 9 for a $4 \times 4$ cluster). Nevertheless, we argue that low-energy two-particle excitations are responsible for transport instead of more common one-particle excitations. As already mentioned above, a detailed discussion of the interaction model will be presented elsewhere.

Once a metallic phase is made plausible for $\mathrm{K}_{3}$ picene compound, the possibility of superconductivity cannot be excluded from a purely theoretical point of view. In this context both many-body effects and electron-phonon interactions would be important [9,14-16,37-40]. Finally, let us remind one that after the seminal work by Mitsuhashi et al. [1] showing the existence of superconducting phases for potassium-intercalated picene, additional experimental evidence points to a metallic behavior of $\mathrm{K}_{3}$ picene. See, for example, the observation of orbital hybridization between picene (C $2 p$ ) and potassium (K 4s) near the Fermi energy obtained by Yamane and Kosugi [41] using soft $\mathrm{x}$-ray spectroscopies or the zero resistivity measurement by Teranishi et al. [7]. Moreover, metallic states in $\mathrm{K}$-intercalated picene films on graphite have been observed very recently [42].

\section{ACKNOWLEDGMENTS}

Financial support by the Spanish MICINN (MAT201126534, CTQ2007-65218, CSD2007-6, FIS2012-33521, FIS2012-35880, and CTQ2011-24165) and the Universidad de Alicante is gratefully acknowledged. We also acknowledge support from the DGUI of the Comunidad de Madrid under the R\&D Program of activities MODELICO-CM/S2009ESP1691.
[1] R. Mitsuhashi, Y. Suzuki, Y. Yamanari, H. Mitamura, T. Kambe, N. Ikeda, H. Okamoto, A. Fujiwara, M. Yamaji, N. Kawasaki, Y. Maniwa, and Y. Kubozono, Nature (London) 464, 76 (2010).

[2] Y. Kubozono, H. Mitamura, X. Lee, X. He, Y. Yamanari, Y. Takahashi, Y. Suzuki, Y. Kaji, R. Eguchi, K. Akaike, T. Kambe, H. Okamoto, A. Fujiwara, T. Kato, T. Kosugi, and H. Aoki, Phys. Chem. Chem. Phys. 13, 16476 (2011).
[3] X. F. Wang, R. H. Liu, Z. Gui, Y. L. Xie, Y. J. Yan, J. J. Ying, X. G. Luo, and X. H. Chen, Nat. Commun. 2, 507 (2011).

[4] M. Q. Xue, T. B. Cao, D. M. Wang, Y. Wu, H. X. Yang, X. L. Dong, J. B. He, F. W. Li, and G. F. Chen, Sci. Rep. 2, 389 (2012).

[5] X. F. Wang, Y. J. Yan, Z. Gui, R. H. Liu, J. J. Ying, X. G. Luo, and X. H. Chen, Phys. Rev. B 84, 214523 (2011). The same group reported enhanced superconductivity by rare-earth-metal doping in phenanthrene (see Ref. [6]). 
[6] X. F. Wang, X. G. Luo, J. J. Ying, Z. J. Xiang, S. L. Zhang, R. R. Zhang, Y. H. Zhang, Y. J. Yan, A. F. Wang, P. Cheng, G. J. Ye, and X. H. Chen, J. Phys.: Condens. Matter 24, 345701 (2012).

[7] K. Teranishi, X. He, Y. Sakai, M. Izumi, H. Goto, R. Eguchi, Y. Takabayashi, T. Kambe, and Y. Kubozono, Phys. Rev. B 87, 060505(R) (2013).

[8] T. Kosugi, T. Miyake, S. Ishibashi, R. Arita, and H. Aoki, J. Phys. Soc. Jpn. 78, 113704 (2009).

[9] G. Giovannetti and M. Capone, Phys. Rev. B. 83, 134508 (2011).

[10] P. L. de Andres, A. Guijarro, and J. A. Vergés, Phys. Rev. B 83, 245113 (2011).

[11] M. Kim, B. I. Min, G. Lee, H. J. Kwon, Y. M. Rhee, and J. H. Shim, Phys. Rev. B 83, 214510 (2011).

[12] T. Kosugi, T. Miyake, S. Ishibashi, R. Arita, and H. Aoki, Phys. Rev. B 84, 214506 (2011).

[13] T. Kambe, X. He, Y. Takahashi, Y. Yamanari, K. Teranishi, H. Mitamura, S. Shibasaki, K. Tomita, R. Eguchi, H. Goto, Y. Takabayashi, T. Kato, A. Fujiwara, T. Kariyado, H. Aoki, and Y. Kubozono, Phys. Rev. B 86, 214507 (2012).

[14] A. Ruff, M. Sing, R. Claessen, H. Lee, M. Tomić, H. O. Jeschke, and R. Valentí, Phys. Rev. Lett. 110, 216403 (2013).

[15] G. H. Zhong, C. Zhang, G. F. Wu, Z. B. Huang, X. J. Chen, and H. Q. Lin, J. Appl. Phys. 113, 17E131 (2013).

[16] J. A. Vergés, P. L. de Andres, E. San-Fabián, G. Chiappe, E. Louis, and A. Guijarro, Phys. Rev. B 85, 165102 (2012).

[17] We think that electrons can hop between separate $K$ groups just because these groups share a picene molecule. The mechanism has been historically named as inner-sphere electron transfer. See, for example, Compendium of Chemical Terminology, 2nd ed. (the "Gold Book"), compiled by A. D. McNaught and A. Wilkinson (Blackwell Scientific Publications, Oxford, 1997). XML on-line corrected version: http://goldbook.iupac.org (2006-), created by M. Nic, J. Jirat, and B. Kosata; updates compiled by A. Jenkins.

[18] Several experimental and theoretical works coincide in positioning $\mathrm{K}$ atoms between intralayer picene molecules in a more or less regular scheme (see Refs. [1,2,8-15]).

[19] M. W. Schmidt, K. K. Baldridge, J. A. Boatz, S. T. Elbert, M. S. Gordon, J. H. Jensen, S. Koseki, N. Matsunaga, K. A. Nguyen, S. J. Su, T. L. Windus, M. Dupuis, and J. A. Montgomery, J. Comput. Chem. 14, 1347 (1993).

[20] M. S. Gordon and M. W. Schmidt, in Theory and Applications of Computational Chemistry, the First Forty Years, edited by C. E. Dykstra, G. Frenking, K. S. Kim, and G. E. Scuseria (Elsevier, Amsterdam, 2005), p. 1167.

[21] A. D. Becke, J. Chem. Phys. 98, 5648 (1993).

[22] C. Lee, W. Yang, and R. G. Parr, Phys. Rev. B 37, 785 (1988).

[23] R. Colle and O. Salvetti, Theor. Chim. Acta 37, 329 (1975).

[24] R. Krishnan, J. S. Binkley, R. Seeger, and J. A. Pople, J. Chem. Phys. 72, 650 (1980).

[25] J.-P. Blaudeau, M. P. McGrath, L. A. Curtiss, and L. Radom, J. Chem. Phys. 107, 5016 (1997).

[26] WXMACMOLPLT package has been employed to represent both the geometry of the studied clusters and the corresponding molecular orbitals. It is described by B. M. Bode and M. S. Gordon, J. Mol. Graphics Mod. 16, 133 (1998).
[27] The calculated separation of $K$ atoms from picene molecule planes is somewhat smaller $(2.60 \AA)$ in our LDA DFT crystal calculation (Ref. [10]). We attribute the discrepancy to the different choice of density functional. Nevertheless, these values are not disturbing both because the structure is still experimentally unknown and because our methodology correctly reproduces experimental values of the $\mathrm{KCH}_{3}$ molecule $(2.67 \AA$ and $1.10 \AA$ for $\mathrm{KC}$ and $\mathrm{CH}$ bonds and $107.6^{\circ}$ for $\mathrm{HCH}$ angle compared to $2.63 \AA, 1.13 \AA$, and $107.0^{\circ}$ measured values). In any case, the full geometrical relaxation of the cluster coordinates is different from a relaxation of the crystal structure with fixed (experimental) cell parameters and may also introduce structural differences between clusters and crystal.

[28] Let us count electrons starting from neutral picene molecules and completely ionized potassium atoms $\left(\mathrm{K}^{+}\right)$. From here on, we will refer to the electrons in this initial electronic configuration as core electrons because their wave functions are very close to those corresponding to isolated molecules or ions. The electrons that are relevant for the transport properties of the compound will be called valence electrons (exactly three per picene molecule assuming a stoichiometric $\mathrm{K}_{3}$ picene compound).

[29] F. Jensen, J. Chem. Phys. 115, 9113 (2001); 116, 7372 (2002); 136, 114107 (2012).

[30] M. Calandra, J. Merino, and R. H. McKenzie, Phys. Rev. B 66, 195102 (2002).

[31] S. Fratini and J. Merino, Phys. Rev. B 80, 165110 (2009).

[32] A. Amaricci, A. Camjayi, K. Haule, G. Kotliar, D. Tanasković, and V. Dobrosavljević, Phys. Rev. B 82, 155102 (2010).

[33] It could be argued that a half-filled Hubbard model for large values of $U$ also shows small energy spin excitations. In this case small energy excitations and insulating behavior appear simultaneously. Nevertheless, the situation seems to be completely different at smaller fillings. In these cases spin excitations are coupled to charge movement and metallic conduction is possible. Later we will use the proposed model to show some illuminating examples. Coming back to the binary compound studied here, it is clear that small energy spin excitations are associated with charge transfer from $\mathrm{K}$ to picene orbitals making a small energy charge transport quite plausible.

[34] R. J. Buenker and S. D. Peyerimhoff, Theor. Chim. Acta 35, 33 (1974).

[35] G. Chiappe, E. Louis, A. Guijarro, E. San-Fabián, and J. A. Vergés, Phys. Rev. B 87, 125126 (2013).

[36] E. Dagotto, Rev. Mod. Phys. 66, 763 (1994).

[37] M. Kim, H. C. Choi, J. H. Shim, and B. I. Min, New J. Phys. 15, 113030 (2013).

[38] A. Subedi and L. Boeri, Phys. Rev. B 84, 020508(R) (2011).

[39] T. Kato, T. Kambe, and Y. Kubozono, Phys. Rev. Lett. 107, 077001 (2011).

[40] M. Casula, M. Calandra, G. Profeta, and F. Mauri, Phys. Rev. Lett. 107, 137006 (2011).

[41] H. Yamane and N. Kosugi, AIP Advances 2, 042114 (2012).

[42] H. Okazaki, T. Jabuchi, T. Wakita, T. Kato, Y. Muraoka, and T. Yokoya, Phys. Rev. B 88, 245414 (2013). 